\title{
Advances and novel developments in environmental influences on allergic diseases
}

\author{
Shifaa Alkotob ${ }^{1}$, Cade Cannedy ${ }^{1}$, Katharina Harter ${ }^{2}$, Hesamaldin Movassagh ${ }^{1}$, Bibek \\ Paudel $^{1}$, Mary Prunicki ${ }^{3}$, Vanitha Sampath ${ }^{3}$, Tamara Schikowski ${ }^{4}$, Eric Smith ${ }^{1}$, Qi Zhao ${ }^{1}$, \\ Claudia Traidl-Hoffmann ${ }^{5}$, and Kari Nadeau ${ }^{1}$ \\ ${ }^{1}$ Stanford University \\ ${ }^{2}$ UNIKA-T \\ ${ }^{3}$ Stanford University School of Medicine \\ ${ }^{4}$ IUF - Leibniz Research Institute for Environmental Medicine \\ ${ }^{5}$ Institute of Environmental Medicine, UNIKA-T
}

June 8, 2020

\begin{abstract}
Atopic diseases have increased in prevalence over the last few decades and the rapid increases suggest that the predominant driving forces behind these increases are environmental factors rather than genetic alterations. A number of environmental factors have been implicated in the increased prevalence of allergic diseases. Predominant among them are increased exposure to pollutants and decreased exposure to microbes and parasitic infections. The hygiene hypothesis suggests that increased hygiene and lack of exposure to microbes and parasitic infections at an early age prevents the necessary stimulus to train the developing immune system to develop tolerogenic responses. Lifestyle factors, such as increased time spent indoors, use of antibiotics, and consumption of processed foods and decreased exposure to farm animals and pets, limit exposure to environmental allergens, infectious parasitic worms, and microbes. The lack of exposure to these factors is thought to prevent proper education and training of the immune system. Other factors that are also associated with increased risk of allergic diseases are Caesarian birth, birth order, tobacco smoke exposure and psychosomatic factors. Here, we review current knowledge on the environmental factors that have been shown to affect the development of allergic diseases and the recent developments in the field.
\end{abstract}

\section{Introduction}

Both genes and the environment shape human health and disease. Although IgE-mediated allergic diseases (atopic diseases) have a genetic component and are more prevalent in individuals with a family history of allergic disease, the observed rapid increases in allergic diseases suggest that environmental factors are the predominant driving forces behind these increases rather than genetic alterations. ${ }^{1,2}$ Common atopic diseases include atopic dermatitis, food allergy, allergic rhinitis, and allergic asthma. Human diets and lifestyle have undergone major alterations. The exposome, which is the sum total of all the exposures of an individual in a lifetime, has undergone major shifts in the last few decades, affecting human health and disease.

A number of factors have been implicated in the increased prevalence of allergic diseases. Predominant among them are increased exposure to pollutants and decreased exposure to microbes and parasitic infections. Air pollution has increased significantly in the last few decades. The hygiene hypothesis suggests that increased hygiene and lack of exposure to microbes and parasitic infections at an early age prevents the necessary stimulus to train the developing immune system to develop tolerogenic responses. Lifestyle factors, such as increased time spent indoors, use of antibiotics, and consumption of processed foods and decreased exposure to farm animals and pets, limit exposure to environmental allergens, infectious parasitic worms, and microbes. 
The lack of exposure to these factors is thought to prevent proper education and training of the immune system. Other factors that are also associated with increased risk of allergic diseases are Caesarian birth, birth order, tobacco smoke exposure and psychosomatic factors.

Increased human population, pollution, and rapid industrialization have affected our environment bringing about climate change. Climate change has led to greater variability in temperature, and increases in air pollution, forest fires, heat waves, droughts, and floods. ${ }^{3}$ Thunderstorms during the pollen season have been linked with increased asthma exacerbations and emergency room visits. ${ }^{4}{ }^{5}$ During thunderstorms, whole pollen grains are swept into the clouds where they are broken up into smaller allergenic pollen fragments and eventually carried back to ground level. ${ }^{6}$ Similarly, dust storms and wildfires have been shown to increase inflammatory responses and asthma exacerbations. ${ }^{7-9}$

A number of recent high-throughput "omic" technologies are accelerating our understanding of allergic diseases and have revolutionized research. The use of the term "omics" suggests a comprehensive highthroughput and systematic investigation of biological parameters. Examples of omic technologies include genomics, epigenomics, transcriptomics, proteomics, metabolomics, microbiomics, and exposomics. ${ }^{10}$ These technologies generate exponentially growing data sets requiring sophisticated bioinformatics and computational techniques that can integrate, analyze and interpret the data to generate hypothesis, which can then be further tested. Of these, epigenomics has been key in giving us insight to gene-environment interactions. It has provided us a greater understanding of the mechanisms by which the environmental factors modulate epigenetic modifications and expression of genes involved in inflammatory responses and allergy. Technologies such as bisulfite sequencing, ATAC seq and cytometry by Time-Of-Flight (EpiTOF) have made it possible to study DNA methylation and histone modifications, and chromatin accessibility across the whole genome and at a single cell level. ${ }^{11-14}$ Here, we review current knowledge on the environmental factors that have been shown to affect the development of allergic diseases and the recent developments in the field.

\section{Factors modulating allergic disease}

Air pollution

Direct effects

Air pollutants considered major risk factors for the development of allergic diseases are ground-level ozone, particulate matter $(\mathrm{PM})$, carbon monoxide $(\mathrm{CO})$, sulfur dioxide $\left(\mathrm{SO}_{2}\right)$, and nitrogen dioxide $\left(\mathrm{NO}_{2}\right) .{ }^{15,} 16$ $\mathrm{CO}, \mathrm{SO}_{2}$, and $\mathrm{NO}_{2}$ are released from combustion of fossil fuels. Ground-level ozone is a secondary pollutant that is produced when nitrogen oxides and volatile organic compounds released from industrial sources react in the presence of sunlight.

PM with diameters [?]10um or smaller (e.g. $\mathrm{PM}_{10}$ and $\mathrm{PM}_{2.5}$ ) can carry organic and inorganic components such as heavy metals and penetrate deeply into the respiratory tract and skin barrier. In a prospective birth cohort study of over 5,000 children during the first 6 years of life, strong positive associations were found between the distance to the nearest main road and asthmatic bronchitis, hay fever, eczema, and sensitization. ${ }^{17}$ An association between eczema and traffic-related pollutants was also found in children from small towns, where exposure to was much lower than in urban areas. ${ }^{18}$ Some studies speculate that ultrafine particles (UFPs) with diameter [?] 100nm may have greater effects due to their increased capacity to penetrate the lung alveoli and cardiovascular system. ${ }^{19} \mathrm{~A}$ recent meta-analysis estimated that increases in UPFs per 10000 particles $/ \mathrm{cm}^{3}$ were associated with 7\%,11\%, and $5 \%$ increase in exacerbations, emergency department visits, and hospital admissions for asthma, respectively. ${ }^{20}$

A recent study estimated that exposure to ambient $\mathrm{NO}_{2}$ may cause 4 million new cases of pediatric asthma per year, with over $60 \%$ occurring in urban areas. ${ }^{21}$ Norbäck et al. observed robust relationships between lifetime exposure to $\mathrm{NO}_{2}$ and allergic diseases including asthma, eczema, wheeze and rhinitis for children ages 3-6 years in China. ${ }^{22}$ Similar associations have been observed with $\mathrm{SO}_{2}$ and $\mathrm{CO}$. A study by Penard-Morand et al. found that $\mathrm{SO}_{2}$ exposure significantly increases the prevalence of asthma in children. ${ }^{23}$ Similarly, Samoli et al. found an association between $\mathrm{SO}_{2}$ and $\mathrm{PM}_{10}$ exposure and the number of pediatric asthma 
hospital admissions among children aged 0 to 14 years in Athens, Greece. ${ }^{24}$ Several time-series studies in China reported positive associations between exposure to $\mathrm{CO}$ within a few days and the risk of hospital admission/mortality from asthma. ${ }^{25}$ Another Korean study found that for children aged 6-7 years, the odds ratio (OR) for life-time allergic rhinitis was 1.10 per $100 \mathrm{ppb}$ increase in $\mathrm{CO}$ concentration during the first year of life. In addition, the OR for current atopic dermatitis was 8.11 for every 1 ppb increase in the average CO concentration during the preceding 12 months. ${ }^{26}$ In the US, the risk for emergency department visits was estimated to increase by $0.8 \%$ for asthma or wheeze and $3.7 \%$ for bronchitis per IQR increase in the preceding 3-day average concentration of $\mathrm{CO} .{ }^{27}$

Ozone in the stratosphere is protective as it shields living things from ultraviolet radiation from the sun. However, ground-level ozone, which forms just above the earth's surface has been associated with adverse health effects. A birth cohort study in Canada reported that ozone exposure at birth was associated with the onset of asthma and allergic rhinitis during a follow-up at age $17 .{ }^{28}$ In France, a higher annual outdoor concentration of ozone was associated with increased total IgE levels. ${ }^{29}$ A study estimated that 7-day exposure to ozone was associated with significant increase in physician visits for atopic dermatitis, contact dermatitis and urticaria. ${ }^{30}$

The pathophysiological mechanisms by which air pollution mediates allergic disease are poorly understood; however, oxidative stress, enhanced sensitization to allergens, inflammatory and immunological responses, and epigenetic modifications have been suggested as possible mechanisms. ${ }^{31-33}$ Exposure of human nasal epithelium cells to $\mathrm{PM}_{2.5}$ was found to decrease loss of barrier function, as determined by measures of transepithelial resistance, permeability, decreased expression of tight junction proteins, and production of proinflammatory cytokines, such as thymic stromal lymphopoietin (TSLP). ${ }^{34}$ A genome-wide DNA methylation study found that long-term ambient air pollution exposure impacts DNA methylation of a number of genes, some of which play a role in inflammatory responses. ${ }^{35}$ Short-term and long-term exposures to high levels of $\mathrm{CO}, \mathrm{NO}_{2}$, and $\mathrm{PM}_{2.5}$ were associated with alterations in differentially methylated regions of Foxp3. ${ }^{36}$

Indirect effect on plants and ecosystems The effects of air pollution reported above on the increase in allergies are direct the immune system or barrier function in humans. However, there is also an indirect effect: air pollution as well as other effects of climate change affect pollen, plants and biodiversity per se. Air pollution (and climate change) affect not only plant growth, pollen and flower production, and duration of the whole pollen season but can also display more indirect health effects by increasing the amount of allergenic encoding transcripts and proteins of the pollen. ${ }^{37,} 38$ When ragweed plants were grown in climate chambers under controlled conditions and fumigated with enhanced levels of $\mathrm{NO}_{2}$, transcript levels of amb were upregulated, indicating potentially higher allergenicity due to $\mathrm{NO}_{2} \cdot{ }^{37}$ On exposure of ragweed to varying $\mathrm{NO}_{2}$ levels during the growing season, a significantly higher allergenicity for Amb a 1 was observed. ${ }^{38}$ Elevated $\mathrm{CO}_{2}$ levels and drought stress was also found to increase allergenic ragweed proteins (Amb a). ${ }^{39}$ Therefore, under global change scenarios the allergenic potential of pollen is also expected to change. Epidemiologic studies have demonstrated that urbanization, high levels of vehicle emissions, and westernized lifestyle are correlated to an increase in the frequency of pollen-induced respiratory allergy prevalent in people who live in urban areas compared to those who live in rural areas - this can in part be due to the effects of pollution on the pollen and plants themselves and therefore indirectly impacting human health.

Tobacco smoke and e-cigarettes

Epidemiological studies and meta-analyses indicate that pre- or post-natal maternal smoking increases the risk of wheezing and asthma in children [?]2 years ${ }^{40}$ and that secondhand smoke during infancy without prior exposure in utero leads to an enhanced risk of food sensitization and eczema. ${ }^{41}$ Tobacco smoke and ecigarettes may mediate their effects via a number of inflammatory mechanisms. For instance, tobacco smoke provokes oxidative stress ${ }^{42}$ which leads to upregulation of TSLP ${ }^{43}$ and IL-33 ${ }^{44}$ suggestive of a pro type-2 inflammation in the lungs. In addition, phagocytic activity of alveolar macrophages from smokers is reduced compared to non-smokers. ${ }^{45}$ Repetitive exposure to cigarette smoke in normal human airway epithelial cells was found to impact the adhesive intercellular junctions and disrupt monolayer integrity. Cortical tension of epithelial cells was observed due to increased actin polymer levels, which further destabilized 
cell adhesion. ${ }^{46}$ Tobacco smoke may also mediate its effect through microbiome dysbiosis. A study found that sensitization to Staphylococcus aureusenterotoxins is increased in smokers with asthma, and it may be a marker of eosinophilic inflammation and severe asthma. ${ }^{45} \mathrm{E}$-cigarettes were also found to be associated with inflammation. A study found that e-cigarette vapors and cigarette smoke altered virulence of key lung pathogens (Haemophilus influenzae, Streptococcus pneumoniae, Staphylococcus aureus and Pseudomonas aeruginosa), which may increase bacterial persistence and inflammatory potential. ${ }^{47}$

\section{Microbiome}

The microbiome has been shown to play a key role in the development of the immune system with microbiome dysbiosis mediating immune deviation. ${ }^{48}$ Characterizing the constituents of the human gastrointestinal, skin, and airway microbiota as well as microbial peptides and metabolites that influence host immunity and immune response to allergens in food allergy, atopic dermatitis, and asthma is the focus of ongoing research. ${ }^{48,}{ }^{49}$ Advances in our understanding of host-microbe interactions have been made possible by $16 \mathrm{~S}$ rRNA sequencing, which permits precise identification and quantification of bacteria. 16S ribosomal RNA gene is a highly conserved locus in the bacteria genome, yet different in sequences among different bacterial species. Another approach is to sequence the total DNA present in one ecosystem using whole genome shotgun techniques, and subsequently map the genes related to microbes, including viruses and fungi. ${ }^{50}$ These techniques have enabled us to make inroads in identifying the species found in a healthy microbiota and those that cause dysbiosis. In atopic dermatitis, $S$ aureus has been shown to be clearly correlated with severity and to decrease during treatment and to rebound after the end of treatment indicating its use as a potential diagnostic and prognostic biomarker. ${ }^{51}$

Studies in mice and humans have shown associations between intestinal bacteria and allergic response to food. In a murine model, germ-free mice were colonized with feces from healthy or cow's milk allergic (CMA) infants. The healthy and CMA mice showed different transcriptome signatures in ileal epithelium, and the healthy mice were protected against anaphylactic responses to cow's milk allergen. The study identified a clostridial species that protected against the allergic response. ${ }^{52}$ Bifidobacterium breve is a species commonly isolated from the intestines of healthy breastfed infants and from human milk and is thought to have a significant impact on the development of immune tolerance. ${ }^{53}$ In a longitudinal study of a Canadian child cohort, it was found that infants at risk of asthma showed gut microbial changes during the first 100 days of life. Four bacteria taxa were reduced in high-risk children and this was accompanied by reduced deregulation of enterohepatic metabolites. To understand causality, the same study also found that inoculating the four taxa of bacteria (Lachnospira, Veillonella, Faecalibacterium, and Rothia) in germ-free mice decreased airway inflammation. ${ }^{54}$ Individuals with atopic dermatitis have reduced skin lipids and increases in Staphylococcus aureus.${ }^{55}$ A study found a correlation between Staphylococcus species-dominated dysbiosis in the skin microbiome and dysregulation of the skin barrier transcriptome in patients with $\mathrm{AD}$, but whether the microbiome dysbiosis is the cause for or result of the skin barrier defect is unclear. ${ }^{56} \mathrm{~S}$. aureus has also been directly correlated with increased expression of inflammatory cytokines, IL-4, IL-13, IL-22, and TSLP and with decreased expression of cathelicidin. ${ }^{57} \mathrm{C}$. difficile colonization during infancy was associated with a higher risk of developing allergic diseases during early childhood. ${ }^{58}$

A number of factors affect the composition of either the skin or gut microbiome. Vaginal delivery, breast feeding, presence of older siblings and exposure to a variety of microorganisms promote healthy microbiota in infants. In contrast, Caesarean section, formula milk, and exposure to antibiotics have a negative impact. ${ }^{59}$ Dietary factors also play a role in microbiome health. Some of these factors are discussed below.

DietIn addition to prebiotics and probiotics, other dietary factors that have been shown to play a role are vitamin D and omega-3 and omega-6 polyunsaturated fatty acids (PUFAs). A study found that higher second trimester n-6 PUFAs were associated with atopic dermatitis in children of women with atopy ${ }^{60} \mathrm{~A}$ meta-analysis found that intake of $\omega-3$ PUFA started during pregnancy may reduce the risk of sensitization to egg and peanut. ${ }^{61}$ Levels of $\omega-3$ and $\omega-6$ were measured in the second trimester and found that higher $\omega-6$ PUFAs were associated with a higher risk of all respiratory outcomes among children if the mother has asthma, but that male children born to women with asthma and a higher PUFA ratio had the highest risk for 
asthma. ${ }^{62}$ A meta-analysis of $\omega-3$ consumption suggests that introduction of fish at 6-9 months and routine consumption once a week reduces asthma and wheeze in children up to 4.5 years old. ${ }^{63}$ The association between vitamin D insufficiency and increased risk of food allergies have been shown by multiple studies. While controlling for regional and population characteristics, places in northern latitudes were found to have more epinephrine autoinjector prescriptions than those in southern latitudes in both USA and Australia. ${ }^{64,} 65$ In another study, food allergies were found to be more likely in infants with low vitamin D. ${ }^{66}$ In children with asthma, vitamin D deficiency was associated with asthma severity and increased serum IgE levels ${ }^{67}$ Farming Environment and Pet Ownership Childhood environments have been shown to play an important role in the protection against allergies. Individuals living at short distances from farms had a lower risk of atopy, as measured by IgE, compared with those living further away. This decrease in atopy risk was even greater for those who grew up on a farm. ${ }^{68}$ Children in rural South African communities with higher exposure to pets and farm animals than children from urban communities were found to be at lower risk of allergic disease. ${ }^{69}$ Marrs et al. reported there was an association between dog ownership at three months of age and protection against food allergies. ${ }^{70}$ However, urban children with pet exposure in the South African cohort had an increased rate of any allergy compared to urban children without pets so conflicting data exists regarding pet ownership in relation to allergies. ${ }^{69}$ Antibiotics Antibiotic usage has been documented to perturb the gut flora of individuals, which places them at an increased risk for the development of allergies and asthma. In mice models of atopic dermatitis, antibiotic use was associated with significantly aggravated phenotypes, including clinical score, transepidermal water loss, and histopathology, compared to those treated with healthy feces or probiotics. ${ }^{71}$ Timing, dose, and frequency of antibiotics in prenatal and infant populations have also been associated with the development of childhood allergies and asthma. ${ }^{72},{ }^{73}$ Short chain fatty acids (SCFAs) which are fermentation end products of insoluble fibers by intestinal microorganisms have been implicated in the maintenance of epithelial integrity and IgA production. ${ }^{74}$ Antibiotics-induced dysbiosis of intestinal microbiota has been shown to increase severity of atopic dermatitis in mice through alterations in SCFA's and decreases in the number of Foxp3+ T regulatory cells. ${ }^{71}$ Vaginal versus caesarean section births The composition of gut flora in children born by caesarean section (C-section) versus vaginal delivery is different and this difference in gut microbiota colonization may impact the development of the immune system. ${ }^{75}$ A vaginal mode of birth exposes the baby to maternal vaginal and fecal flora. ${ }^{76}$ Studies indicate that babies born via C-section have a higher incidence of allergy, atopy, and asthma, increased susceptibility to infectious wheezing ${ }^{77}$ and decreased gut microbiome diversity. ${ }^{78}$ In addition, long-term studies show greater incidence of childhood asthma up to the age of 12 years. ${ }^{79}$ Other factorsIn addition to environmental and lifestyle factors, household composition has also been shown to affect the risk of allergic diseases. A study that followed 17,414 British children for 23 years found a strong association between the birth order of a child and the risk of hay fever. ${ }^{80}$ Specifically, contact with older siblings was hypothesized to increase immunological protection due to an increase in infections in early childhood through unhygienic contacts with siblings. A study on 10,834 children enrolled in the Chicago Family Cohort Food Allergy study found that younger siblings of kids with food allergies had significantly less prevalence of food sensitization and clinical food allergy. ${ }^{81}$ Current research shows that psychosocial stress and poor mental health in mothers increase the risk of allergic diseases in their children. ${ }^{82-88}$ Stressful life events in childhood, for example parental divorce, have also been shown to increase the risk for development of atopic eczema later in life. ${ }^{89}$ Psychosocial stress might trigger or worsen allergic symptoms ${ }^{90}$ Also, in adults with allergies, psychoneuroimmunologic mechanisms might play an important role. ${ }^{91}$ An association of anxiety and depression with allergies was reported in many studies. $^{92,}, 93$

\section{Summary and Future Directions}

There have been exciting new developments in understanding the role of the environment in mediating allergic diseases. Epigenetics has provided us better insight into how pollution and other environmental factors alter gene expression. Novel high throughput technologies are enabling characterization of a healthy microbiota and the imbalance that is created with microbial dysbiosis. This knowledge can assist with preventative strategies that can restore a healthy microbiota. It is now recognized that early infancy offers a "critical window" of colonization during which microbial communities shape immune maturation and this 
window may enable opportunities for preventative treatments with pre- and probiotics. ${ }^{94}$ In humans, this critical period appears to be within the first 100 days of life. ${ }^{95}$ A study found that supplementing infants with a probiotic mixture together with at least partial breastfeeding corrected undesired changes in microbiota composition and function caused by antibiotic treatments or caesarean birth. ${ }^{96}$ Host microbe interactions are highly complex and their role in medicating allergy and asthma continues to be an area of intense research. ${ }^{97}$ Development of tolerance towards food allergens also appears to occur at an early age. A large study, the Learning Early About Peanuts (LEAP) study, found that the early introduction of peanuts significantly decreased the frequency of the development of peanut allergy among children at high risk for this allergy. ${ }^{98}$ Current guidelines for allergy prevention now encourage active introduction of allergenic foods to all infants from 4-6 months of age. ${ }^{99}$

Atopic diseases have common underlying mechanisms. Epidemiological studies show a natural history for the progression of these diseases, starting with atopic dermatitis in early infancy and progressing to food allergy, allergic rhinitis, and allergic asthma. This natural progression of atopic diseases is termed the Atopic March. Studies are now evaluating if by prevention of atopic dermatitis, we can prevent the subsequent manifestation of other atopic diseases. In addition to the development of targeted therapeutics, studies are determining if the use of emollients to treat skin barrier disruption can prevent atopic dermatitis and other atopic diseases. ${ }^{100}$

Ultimately understanding the role that genes, epigenetics, and the environment play in shaping our immune health at the DNA, RNA, and protein level is key to developing targeted therapies for preventing and treating allergic diseases.

\section{REFERENCES}

1. Doll R.J., Joseph N.I., McGarry D., Jhaveri D., Sher T., R. H. Epidemiology of Allergic Diseases. . In: M M, editor. Allergy and Asthma. Cham: Springer; 2019.

2. Gilles S, Akdis C, Lauener R, et al. The role of environmental factors in allergy: A critical reappraisal. Exp Dermatol2018;27 :1193-1200.

3. Intergovernmental panel on climate change. Climate Change 2013: The Physical Science Basis. Available from: https://www.ipcc.ch/site/assets/uploads/2018/03/WG1AR5_SummaryVolume_FINAL.pdf. Accessed May 15, 2020

4. Harun NS, Lachapelle P, Douglass J. Thunderstorm-triggered asthma: what we know so far. J Asthma Allergy 2019;12:101-108.

5. Woodhead M. Hospitals overwhelmed with patients after "thunderstorm asthma" hits Melbourne. BMJ 2016;355 :i6391.

6. D'Amato G, Vitale C, D'Amato M, et al. Thunderstorm-related asthma: what happens and why. Clin Exp Allergy 2016;46:390-396.

7. James KA, Strand M, Hamer MK, Cicutto L. Health Services Utilization in Asthma Exacerbations and PM10 Levels in Rural Colorado. Ann Am Thorac Soc 2018;15 :947-954.

8. Reid CE, Jerrett M, Tager IB, Petersen ML, Mann JK, Balmes JR. Differential respiratory health effects from the 2008 northern California wildfires: A spatiotemporal approach. Environ Res2016;150 :227-235.

9. Prunicki MM, Dant CC, Cao S, et al. Immunologic effects of forest fire exposure show increases in IL-1beta and CRP. Allergy 2020. DOI: 10.1111/all.14251.

10. Donovan BM, Bastarache L, Turi KN, Zutter MM, Hartert TV. The current state of omics technologies in the clinical management of asthma and allergic diseases. Ann Allergy Asthma Immunol2019;123:550-557.

11. Buenrostro JD, Wu B, Chang HY, Greenleaf WJ. ATAC-seq: A Method for Assaying Chromatin Accessibility Genome-Wide. Curr Protoc Mol Biol 2015;109 :21 29 21-21 2929. 
12. Schwartzman O, Tanay A. Single-cell epigenomics: techniques and emerging applications. Nat Rev Genet 2015;16 :716-726.

13. Cheung P, Vallania F, Warsinske HC, et al. Single-Cell Chromatin Modification Profiling Reveals Increased Epigenetic Variations with Aging. Cell 2018;173 :1385-1397 e1314.

14. Forno E, Wang T, Qi C, et al. DNA methylation in nasal epithelium, atopy, and atopic asthma in children: a genome-wide study. Lancet Respir Med 2019;7 :336-346.

15. United States Enviromental Protectiion Agency. Criteria Air Pollutants. 2017 Available from: https://www.epa.gov/criteria-air-pollutants\#self. Accessed May 15, 2020

16. World Health Organization. Ambient air pollution: Pollutants. 2020 Available from: https://www.who.int/airpollution/ambient/pollutants/en/. Accessed May 15, 2020

17. Morgenstern V, Zutavern A, Cyrys J, et al. Atopic diseases, allergic sensitization, and exposure to traffic-related air pollution in children. Am J Respir Crit Care Med 2008;177 :1331-1337.

18. Kramer U, Sugiri D, Ranft U, et al. Eczema, respiratory allergies, and traffic-related air pollution in birth cohorts from small-town areas. J Dermatol Sci 2009;56 :99-105.

19. Ohlwein S, Kappeler R, Kutlar Joss M, Kunzli N, Hoffmann B. Health effects of ultrafine particles: a systematic literature review update of epidemiological evidence. Int J Public Health2019;64 :547-559.

20. Li Q, Yi Q, Tang L, et al. Influence of Ultrafine Particles Exposure on Asthma Exacerbation in Children: A Meta-Analysis. Curr Drug Targets 2019;20 :412-420.

21. Achakulwisut P, Brauer M, Hystad P, Anenberg SC. Global, national, and urban burdens of paediatric asthma incidence attributable to ambient NO2 pollution: estimates from global datasets. Lancet Planet Health 2019;3 :e166-e178.

22. Norbäck D, Lu C, Zhang Y, et al. Sources of indoor particulate matter (PM) and outdoor air pollution in China in relation to asthma, wheeze, rhinitis and eczema among pre-school children: Synergistic effects between antibiotics use and PM10 and second hand smoke.Environment international 2019;125 :252-260.

23. Penard-Morand C, Raherison C, Charpin D, et al. Long-term exposure to close-proximity air pollution and asthma and allergies in urban children. Eur Respir J 2010;36 :33-40.

24. Samoli E, Nastos PT, Paliatsos AG, Katsouyanni K, Priftis KN. Acute effects of air pollution on pediatric asthma exacerbation: evidence of association and effect modification. Environ Res2011;111:418-424.

25. Zhao Y, Hu J, Tan Z, et al. Ambient carbon monoxide and increased risk of daily hospital outpatient visits for respiratory diseases in Dongguan, China. Sci Total Environ 2019;668 :254-260.

26. Kim J, Han Y, Seo SC, et al. Association of carbon monoxide levels with allergic diseases in children. Allergy Asthma Proc2016;37 :e1-7.

27. Xiao Q, Liu Y, Mulholland JA, et al. Pediatric emergency department visits and ambient Air pollution in the U.S. State of Georgia: a case-crossover study. Environ Health 2016;15 :115.

28. To T, Zhu J, Stieb D, et al. Early life exposure to air pollution and incidence of childhood asthma, allergic rhinitis and eczema.Eur Respir J 2020;55 .

29. Rage E, Jacquemin B, Nadif R, et al. Total serum IgE levels are associated with ambient ozone concentration in asthmatic adults. Allergy 2009;64:40-46.

30. Valacchi G, Fortino V, Bocci V. The dual action of ozone on the skin. Br J Dermatol 2005;153 :1096-1100.

31. Brandt EB, Biagini Myers JM, Acciani TH, et al. Exposure to allergen and diesel exhaust particles potentiates secondary allergen-specific memory responses, promoting asthma susceptibility. J Allergy Clin Immunol 2015;136 :295-303 e297. 
32. Munoz X, Barreiro E, Bustamante V, Lopez-Campos JL, Gonzalez-Barcala FJ, Cruz MJ. Diesel exhausts particles: Their role in increasing the incidence of asthma. Reviewing the evidence of a causal link. Sci Total Environ 2019;652:1129-1138.

33. Naclerio R, Ansotegui IJ, Bousquet J, et al. International expert consensus on the management of allergic rhinitis (AR) aggravated by air pollutants: Impact of air pollution on patients with AR: Current knowledge and future strategies. World Allergy Organ J2020;13:100106.

34. Xian M, Ma S, Wang K, et al. Particulate Matter 2.5 Causes Deficiency in Barrier Integrity in Human Nasal Epithelial Cells.Allergy Asthma Immunol Res 2020;12 :56-71.

35. Lee MK, Xu CJ, Carnes MU, et al. Genome-wide DNA methylation and long-term ambient air pollution exposure in Korean adults. Clin Epigenetics 2019;11 :37.

36. Prunicki M, Stell L, Dinakarpandian D, et al. Exposure to NO2, CO, and PM2.5 is linked to regional DNA methylation differences in asthma. Clin Epigenetics 2018;10 :2.

37. Zhao F, Durner J, Winkler JB, et al. Pollen of common ragweed (Ambrosia artemisiifolia L.): Illuminabased de novo sequencing and differential transcript expression upon elevated $\mathrm{NO}(2) / \mathrm{O}(3)$. Environ Pollut 2017;224:503-514.

38. Zhao F, Elkelish A, Durner J, et al. Common ragweed (Ambrosia artemisiifolia L.): allergenicity and molecular characterization of pollen after plant exposure to elevated NO2. Plant Cell Environ2016;39:147164.

39. El Kelish A, Zhao F, Heller W, et al. Ragweed (Ambrosia artemisiifolia) pollen allergenicity: SuperSAGE transcriptomic analysis upon elevated CO2 and drought stress. BMC Plant Biol2014;14:176.

40. Burke H, Leonardi-Bee J, Hashim A, et al. Prenatal and passive smoke exposure and incidence of asthma and wheeze: systematic review and meta-analysis. Pediatrics 2012;129 :735-744.

41. Thacher JD, Gruzieva O, Pershagen G, et al. Parental smoking and development of allergic sensitization from birth to adolescence.Allergy 2016;71 :239-248.

42. Chao MR, Cooke MS, Kuo CY, et al. Children are particularly vulnerable to environmental tobacco smoke exposure: Evidence from biomarkers of tobacco-specific nitrosamines, and oxidative stress.Environ Int 2018;120 :238-245.

43. Nakamura Y, Miyata M, Ohba T, et al. Cigarette smoke extract induces thymic stromal lymphopoietin expression, leading to $\mathrm{T}(\mathrm{H}) 2$-type immune responses and airway inflammation. J Allergy Clin Immunol2008;122:1208-1214.

44. Kearley J, Silver JS, Sanden C, et al. Cigarette smoke silences innate lymphoid cell function and facilitates an exacerbated type I interleukin-33-dependent response to infection. Immunity2015;42 :566-579.

45. Strzelak A, Ratajczak A, Adamiec A, Feleszko W. Tobacco Smoke Induces and Alters Immune Responses in the Lung Triggering Inflammation, Allergy, Asthma and Other Lung Diseases: A Mechanistic Review. Int J Environ Res Public Health 2018;15.

46. Nishida K, Brune KA, Putcha N, et al. Cigarette smoke disrupts monolayer integrity by altering epithelial cell-cell adhesion and cortical tension. Am J Physiol Lung Cell Mol Physiol2017;313 :L581-L591.

47. Gilpin DF, McGown KA, Gallagher K, et al. Electronic cigarette vapour increases virulence and inflammatory potential of respiratory pathogens. Respir Res 2019;20:267.

48. Peroni DG, Nuzzi G, Trambusti I, Di Cicco ME, Comberiati P. Microbiome Composition and Its Impact on the Development of Allergic Diseases. Front Immunol 2020;11:700.

49. Borbet TC, Zhang X, Muller A, Blaser MJ. The role of the changing human microbiome in the asthma pandemic. J Allergy Clin Immunol2019;144 :1457-1466. 
50. Ranjan R, Rani A, Metwally A, McGee HS, Perkins DL. Analysis of the microbiome: Advantages of whole genome shotgun versus 16S amplicon sequencing. Biochem Biophys Res Commun 2016;469 :967-977.

51. Reiger M, Traidl-Hoffmann C, Neumann AU. The skin microbiome as a clinical biomarker in atopic eczema: Promises, navigation, and pitfalls.J Allergy Clin Immunol 2020;145:93-96.

52. Feehley T, Plunkett CH, Bao R, et al. Healthy infants harbor intestinal bacteria that protect against food allergy. Nat Med2019;25:448-453.

53. Cukrowska B, Bierla JB, Zakrzewska M, Klukowski M, Maciorkowska E. The Relationship between the Infant Gut Microbiota and Allergy. The Role of Bifidobacterium breve and Prebiotic Oligosaccharides in the Activation of Anti-Allergic Mechanisms in Early Life. Nutrients2020;12 .

54. Arrieta MC, Stiemsma LT, Dimitriu PA, et al. Early infancy microbial and metabolic alterations affect risk of childhood asthma. Sci Transl Med 2015;7 :307ra152.

55. Lipsky ZW, Marques CNH, German GK. Lipid depletion enables permeation of Staphylococcus aureus bacteria through human stratum corneum. Tissue Barriers 2020:1754706.

56. Altunbulakli C, Reiger M, Neumann AU, et al. Relations between epidermal barrier dysregulation and Staphylococcus species-dominated microbiome dysbiosis in patients with atopic dermatitis. J Allergy Clin Immunol 2018;142 :1643-1647 e1612.

57. Nakatsuji T, Chen TH, Two AM, et al. Staphylococcus aureus Exploits Epidermal Barrier Defects in Atopic Dermatitis to Trigger Cytokine Expression. J Invest Dermatol 2016;136 :2192-2200.

58. Lee SH, Gong YN, Ryoo E. Clostridium difficile colonization and/or infection during infancy and the risk of childhood allergic diseases.Korean J Pediatr 2017;60 :145-150.

59. Mohajeri MH, Brummer RJM, Rastall RA, et al. The role of the microbiome for human health: from basic science to clinical applications. Eur J Nutr 2018;57 :1-14.

60. Gardner KG, Gebretsadik T, Hartman TJ, et al. Prenatal Omega-3 and Omega-6 Polyunsaturated Fatty Acids and Childhood Atopic Dermatitis.J Allergy Clin Immunol Pract 2020;8 :937-944.

61. Vahdaninia M, Mackenzie H, Dean T, Helps S. omega-3 LCPUFA supplementation during pregnancy and risk of allergic outcomes or sensitization in offspring: A systematic review and meta-analysis.Ann Allergy Asthma Immunol 2019;122 :302-313 e302.

62. Rosa MJ, Hartman TJ, Adgent M, et al. Prenatal polyunsaturated fatty acids and child asthma: Effect modification by maternal asthma and child sex. J Allergy Clin Immunol 2020;145:800-807 e804.

63. Papamichael MM, Shrestha SK, Itsiopoulos C, Erbas B. The role of fish intake on asthma in children: A meta-analysis of observational studies. Pediatr Allergy Immunol 2018;29 :350-360.

64. Camargo CA, Jr., Clark S, Kaplan MS, Lieberman P, Wood RA. Regional differences in EpiPen prescriptions in the United States: the potential role of vitamin D. J Allergy Clin Immunol2007;120 :131-136.

65. Mullins RJ, Clark S, Camargo CA, Jr. Regional variation in epinephrine autoinjector prescriptions in Australia: more evidence for the vitamin D-anaphylaxis hypothesis. Ann Allergy Asthma Immunol2009;103 :488-495.

66. Allen KJ, Koplin JJ, Ponsonby AL, et al. Vitamin D insufficiency is associated with challenge-proven food allergy in infants. J Allergy Clin Immunol 2013;131 :1109-1116, 1116 e1101-1106.

67. Mohammadzadeh I, Darvish S, Qujeq D, Hajiahmadi M, Vaghari-Tabari M. Association of serum 25-OH vitamin $\mathrm{D}(3)$ with serum IgE and the Pediatric Asthma Severity Score in patients with pediatric asthma. Allergy Asthma Proc 2020;41:126-133. 
68. Borlee F, Yzermans CJ, Krop EJM, et al. Residential proximity to livestock farms is associated with a lower prevalence of atopy. Occup Environ Med 2018;75:453-460.

69. Levin ME, Botha M, Basera W, et al. Environmental factors associated with allergy in urban and rural children from the South African Food Allergy (SAFFA) cohort. J Allergy Clin Immunol2020;145 :415-426.

70. Marrs T, Logan K, Craven J, et al. Dog ownership at three months of age is associated with protection against food allergy. Allergy2019;74:2212-2219.

71. Kim HJ, Lee SH, Hong SJ. Antibiotics-Induced Dysbiosis of Intestinal Microbiota Aggravates Atopic Dermatitis in Mice by Altered Short-Chain Fatty Acids. Allergy Asthma Immunol Res 2020;12 :137-148.

72. Zven SE, Susi A, Mitre E, Nylund CM. Association Between Use of Multiple Classes of IAntibiotic in Infancy and Allergic Disease in Childhood. JAMA Pediatr. 2019;174 (2):199-200.

73. Mitre E, Susi A, Kropp LE, Schwartz DJ, Gorman GH, Nylund CM. Association Between Use of AcidSuppressive Medications and Antibiotics During Infancy and Allergic Diseases in Early Childhood. JAMA Pediatr 2018;172 :e180315.

74. O’Mahony L. Short Chain Fatty Acids Modulate Mast Cell Activation.Allergy 2020.

75. Dominguez-Bello MG, Costello EK, Contreras M, et al. Delivery mode shapes the acquisition and structure of the initial microbiota across multiple body habitats in newborns. Proc Natl Acad Sci U S A2010;107 :11971-11975.

76. Stokholm J, Thorsen J, Chawes BL, et al. Cesarean section changes neonatal gut colonization. J Allergy Clin Immunol2016;138:881-889 e882.

77. Higgins D, Karmaus W, Jiang Y, Banerjee P, Sulaiman IM, Arshad HS. Infant wheezing and prenatal antibiotic exposure and mode of delivery: a prospective birth cohort study. $J$ Asthma 2020:1-12.

78. Sandall J, Tribe RM, Avery L, et al. Short-term and long-term effects of caesarean section on the health of women and children. Lancet 2018;392:1349-1357.

79. Keag OE, Norman JE, Stock SJ. Long-term risks and benefits associated with cesarean delivery for mother, baby, and subsequent pregnancies: Systematic review and meta-analysis. PLoS Med2018;15 :e1002494.

80. Strachan DP. Hay fever, hygiene, and household size. BMJ1989;299:1259-1260.

81. Gupta RS, Walkner MM, Greenhawt M, et al. Food Allergy Sensitization and Presentation in Siblings of Food Allergic Children. J Allergy Clin Immunol Pract 2016;4 :956-962.

82. Brew BK, Lundholm C, Viktorin A, Lichtenstein P, Larsson H, Almqvist C. Longitudinal depression or anxiety in mothers and offspring asthma: a Swedish population-based study. Int J Epidemiol2018;47 :166-174.

83. Smejda K, Polanska K, Merecz-Kot D, et al. Maternal Stress During Pregnancy and Allergic Diseases in Children During the First Year of Life. Respir Care 2018;63 :70-76.

84. Magnus MC, Wright RJ, Roysamb E, et al. Association of Maternal Psychosocial Stress With Increased Risk of Asthma Development in Offspring. Am J Epidemiol 2018;187 :1199-1209.

85. Flanigan C, Sheikh A, DunnGalvin A, Brew BK, Almqvist C, Nwaru BI. Prenatal maternal psychosocial stress and offspring's asthma and allergic disease: A systematic review and meta-analysis. Clin Exp Allergy $2018 ; 48: 403-414$.

86. Rosa MJ, Lee AG, Wright RJ. Evidence establishing a link between prenatal and early-life stress and asthma development. Curr Opin Allergy Clin Immunol 2018;18:148-158.

87. Chan CWH, Law BMH, Liu YH, et al. The Association between Maternal Stress and Childhood Eczema: A Systematic Review. Int J Environ Res Public Health 2018;15 . 
88. Braig S, Weiss JM, Stalder T, Kirschbaum C, Rothenbacher D, Genuneit J. Maternal prenatal stress and child atopic dermatitis up to age 2 years: The Ulm SPATZ health study. Pediatr Allergy Immunol2017;28 $: 144-151$.

89. Herberth G, Roder S, Bockelbrink A, et al. Stressful life events in childhood and allergic sensitization. Allergol Select2018;2:1-9.

90. Patterson AM, Yildiz VO, Klatt MD, Malarkey WB. Perceived stress predicts allergy flares. Ann Allergy Asthma Immunol2014;112:317-321.

91. Marshall GD, Tull MT. Stress, mindfulness, and the allergic patient.Expert Rev Clin Immunol 2018;14 :1065-1079.

92. Harter K, Hammel G, Krabiell L, et al. Different Psychosocial Factors Are Associated with Seasonal and Perennial Allergies in Adults: Cross-Sectional Results of the KORA FF4 Study. Int Arch Allergy Immunol $2019 ; 179: 262-272$.

93. Ronnstad ATM, Halling-Overgaard AS, Hamann CR, Skov L, Egeberg A, Thyssen JP. Association of atopic dermatitis with depression, anxiety, and suicidal ideation in children and adults: A systematic review and meta-analysis. J Am Acad Dermatol 2018;79 :448-456 e430.

94. Ver Heul A, Planer J, Kau AL. The Human Microbiota and Asthma.Clin Rev Allergy Immunol 2019;57 :350-363.

95. Stiemsma LT, Turvey SE. Asthma and the microbiome: defining the critical window in early life. Allergy Asthma Clin Immunol2017;13:3.

96. Korpela K, Salonen A, Vepsalainen O, et al. Probiotic supplementation restores normal microbiota composition and function in antibiotic-treated and in caesarean-born infants. Microbiome2018;6 :182.

97. Salameh M, Burney Z, Mhaimeed N, et al. The role of gut microbiota in atopic asthma and allergy, implications in the understanding of disease pathogenesis. Scand J Immunol 2020;91 :e12855.

98. Turcanu V, Brough HA, Du Toit G, et al. Immune mechanisms of food allergy and its prevention by early intervention. Curr Opin Immunol 2017;48:92-98.

99. Baker MG, Nowak-Wegrzyn A. Food allergy prevention: current evidence. Curr Opin Clin Nutr Metab Care 2020;23 :196-202.

100. Brough HA, Nadeau KC, Sindher SB, et al. Epicutaneous sensitization in the development of food allergy: What is the evidence and how can this be prevented? Allergy 2020. DOI: 10.1111/all.14304.

Box 1:

- Characterization of microbes in healthy and allergic individuals

- Identifying type and optimal timing of introduction of probiotics and prebiotics for tolerance induction

- Identifying epigenetic alterations and changes in gene expression on exposure to pollutants

- Evaluating emollients as treatment for prevention of atopic dermatitis and other atopic disorders.

- Targeted therapy for precision medicine

Figure 1 


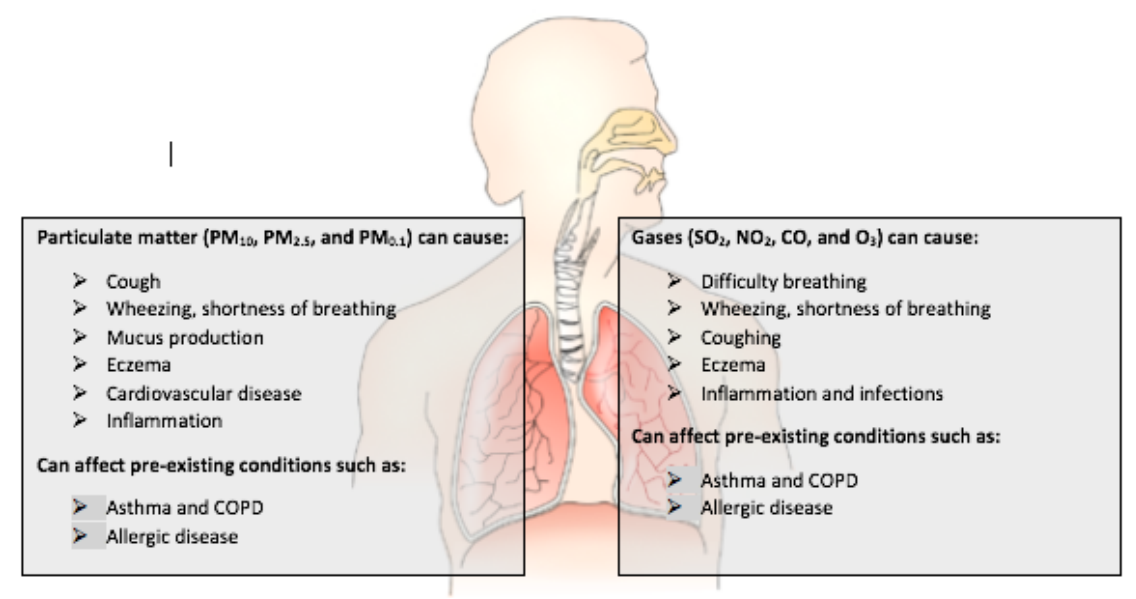

Figure 1: Air pollution affects risk of allergic disease. Air pollutants include particulate matter (PM) and gases. Particulate matter that cause allergic disease include PM with diameters [?]10um or smaller (e.g. $\mathrm{PM}_{10}$ and $\mathrm{PM}_{2.5}$ ). Major gaseous pollutants that affect include $\mathrm{SO}_{2}, \mathrm{NO}_{2}, \mathrm{CO}, \mathrm{O}_{3}$.

\section{Figure 2}
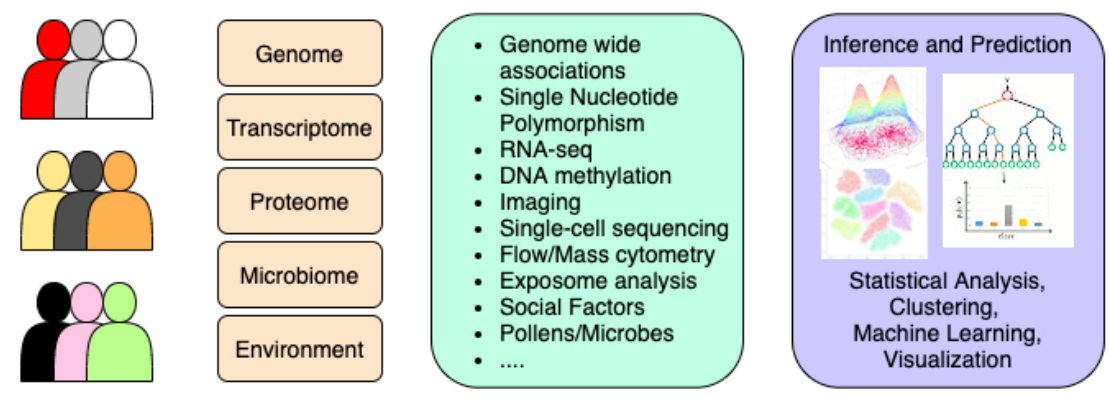

Figure 2: High throughput omics technologies enable generation of large amounts of data that are analyzed and interpreted by sophisticated bioinformatics and computational tools to give us mechanistic information on immune pathways at the DNA, RNA, and protein level. 